\title{
Materialität und Macht im Spiegel der Assemblage-Theorie: Erkundungen am Beispiel der Waldpolitik in Thailland
}

\author{
A. Mattissek ${ }^{1}$ and T. Wiertz ${ }^{2}$ \\ ${ }^{1}$ Institut für Geographie, Technische Universität Dresden, Germany \\ ${ }^{2}$ Institute for Advanced Sustainabilitiy Studies e.V. (IASS), Potsdam, Germany \\ Correspondence to: T. Wiertz (thilo.wiertz@iass-potsdam.de)
}

Received: 22 February 2013 - Revised: 23 July 2013 - Accepted: 7 August 2013 - Published: 8 October 2014

\begin{abstract}
Kurzfassung. Nature and technology are at the core of many ongoing social transformations and political struggles. While constructivist approaches in general and poststructuralist theories in particular point to the discursive negotiation of materiality, they have so far failed to adequately account for its constitutive role in stabilizing and destabilizing social relations. We argue that theories based on a "flat ontology" offer a way to re-materialize social theory while keeping the sensitivity to power-knowledge relations that poststructuralist theories have developed. Drawing on the work of Deleuze and Guattari and recent discussions on Assemblage Theory in Human Geography, we sketch out a theoretical framework that conceptualizes the relations between symbolic and material entities in a non-deterministic way. Using the example of recent shifts in forest politics in Thailand under the influence of climate change policies, we discuss some of the empirical aspects that can be analyzed with the help of Assemblage Theory.
\end{abstract}

\section{Einleitung}

Der Wandel politischer Ordnungen ist immer auch an physische, biologische oder technische Veränderungen geknüpft, wie bereits ein flüchtiger Blick auf aktuelle gesellschaftliche Debatten zeigt. Der internationale Konflikt um das iranische Atomprogramm, die Energiewende in Deutschland, Ernährungsprobleme einer wachsenden Weltbevölkerung, Proteste gegen die Einlagerung von Kohlenstoffdioxid in geologischen Lagerstätten oder Strategien im Kampf gegen globale Gesundheitsrisiken sind nur einige Beispiele dafür, dass „Materialität“ kein Außen politischer Auseinandersetzungen bildet, sondern untrennbar mit ihnen verstrickt ist. Diese Einsicht spiegelt sich in Theoriediskussionen wider, die seit einiger Zeit in den Sozialwissenschaften und in der Geographie geführt werden und auf die materielle Verfasstheit von Gesellschaft verweisen. Dabei geht es sowohl um das konzeptionelle Verhältnis des Zusammenhangs symbolischer und materieller Aspekte gesellschaftlicher Wirklichkeit als auch um Implikationen für die empirische Forschung.
Arbeiten der Neuen Kulturgeographie und der Politischen Geographie, die sich auf poststrukturalistische Theorien berufen, heben die „Gemachtheit“ gesellschaftlicher Welten und ihre prinzipielle Veränderlichkeit hervor, indem sie zum Beispiel symbolische Aushandlungsprozesse um Natur und Technik in den Blick nehmen. Sie zeigen, dass Materialität gesellschaftliche Verhältnisse nicht determiniert, sondern als Gegenstand von Diskursen selbst einer Erklärung bedarf. Indem sie symbolische Ordnungen losgelöst von einem nicht-diskursiven Außen betrachten, rücken sie die Machtverhältnisse bei der Konstitution und Veränderung gesellschaftlicher Ordnungen in das Blickfeld. Eine solche Loslösung kann jedoch auch zu einem „Realitätsverlust“ führen, insofern sich diskurstheoretische Ansätze häufig gleichgültig gegenüber der materiellen Dimension von Gesellschaft zeigen. Dieser blinde Fleck hat eine Debatte um die ReMaterialisierung humangeographischer Forschung nach dem cultural turn provoziert (Whatmore, 2006; Jackson, 2000; Philo, 2000; Kazig und Weichhart, 2009). Viele aktuelle Arbeiten aus unterschiedlichen Teilbereichen einer konstruktivistisch informierten Humangeographie schenken daher 
beispielsweise der Dynamik der physischen Umwelt oder der Körperlichkeit sozialer Praktiken wieder erhöhte Aufmerksamkeit (Braun, 2008).

Geht es um die kritische Erklärung der Entstehung und des Wandels gesellschaftlicher Ordnungen im Grenzbereich von Diskurs und Materialität, so erscheint uns eine junge Debatte um Flache Ontologien und Assemblage-Theorien vielversprechend, die ihre Inspiration aus den philosophischen Arbeiten von Gilles Deleuze und Felix Guattari bezieht (Marston et al., 2005; Escobar, 2007; Anderson et al., 2012). Die Attraktivität dieser Debatte liegt unseres Erachtens darin, dass sie es erlaubt, kritische Erklärungen sozialer Wirklichkeiten um die Dimension des Materiellen zu erweitern und gleichzeitig zentrale Prämissen politischgeographischer Forschung beizubehalten: die Abkehr von essentialistischem und deterministischem Denken und die Betonung von Machtverhältnissen. Insbesondere die Schnittmengen mit Foucaults Arbeiten zur Gouvernementalität und seinem Begriff des Dispositivs (Legg, 2011) bieten die Möglichkeit, die materiellen Facetten eines poststrukturalistischen Machtkonzepts hervorzuheben und den Zwischenraum symbolischer und materieller Ordnungen zum Schauplatz geographischer Forschung zu machen.

Wir werden im Folgenden zunächst die Rolle von Materialität in poststrukturalistischen Ansätzen skizzieren, um dann Kerngedanken einer Theorie darzustellen, die Agencements zum Ausgangspunkt für sozialwissenschaftliche und geographische Forschung macht. Das Konzept des Agencement verweist auf Verknüpfungen heterogener Elemente, in denen unterschiedliche Logiken zusammenwirken und soziomaterielle Wirklichkeiten stabilisieren und destabilisieren. Deleuze und Guattari (1992) bieten dabei auch ein Verständnis des Verhältnisses von Sprache und Materialität an, das wir im vierten Abschnitt diskutieren. Wir verweisen auf mögliche Implikationen für die Humangeographie sowie für ihren Zugang zu gesellschaftlichen Machtverhältnissen und erkunden einige Möglichkeiten des Konzepts am Beispiel aktueller Transformationen in der Waldpolitik in Thailand.

\section{Materialität in poststrukturalistischen Ansätzen}

Poststrukturalistische Ansätze innerhalb der Humangeographie sind als Gegenbewegung zu essentialistischen Kulturund Subjektbegriffen sowie zu geo- und raumdeterministischen Erklärungen von Gesellschaft zu sehen. Anstatt nach universellen Gesetzmäßigkeiten des Gesellschaftlichen zu suchen, betonen sie, dass gesellschaftliche Stabilisierungen und Veränderungen als Ergebnis von Bedeutungszuschreibungen zu verstehen sind. Themenfelder der Neuen Kulturgeographie, der Gesellschaft-Umwelt-Forschung und der Politischen Geographie treten dann unter dem Gesichtspunkt hervor, wie sich das Verhältnis von Macht und Wissen beispielsweise in geopolitischen Leitbildern, in der Konfrontation des „Eigenen“ mit dem „Fremden“, in unterschied- lichen Naturverständnissen und in spezifischen Denk- und Handlungslogiken niederschlägt. Aufgabe der Forschung ist es, die in gesellschaftlichen Beziehungen eingeschriebenen Machtverhältnisse aufzuzeigen und sie somit der Kritik zugänglich zu machen. Der Anspruch einer poststrukturalistischen Perspektive lässt sich also als eine kritische Erklärung der Stabilisierung und Veränderung gesellschaftlicher Wirklichkeiten verstehen, mit dem Ziel, Ordnung und Wandel auf den Aspekt ihrer symbolischen Gemachtheit - und damit prinzipiellen Veränderlichkeit - zu untersuchen (Glynos und Howarth, 2007). Die Operationalisierung diskurstheoretischer und poststrukturalistischer Ansätze ist mittlerweile in einer Vielzahl empirischer Arbeiten diskutiert und erprobt worden (Glasze und Mattissek, 2009; Keller et al., 2011).

Der erkenntnistheoretische Konstruktivismus, der poststrukturalistischen Ansätzen zu Grunde liegt, ist als strategische Denkbewegung zu sehen; als Reaktion auf die Unzulänglichkeiten linear-kausaler und deterministischer Erklärungsmodelle, die realistische bzw. materialistische Theorien häufig kennzeichnen, und die Verquickung solcher Modelle mit politischen Machtverhältnissen. Zwar treten durch die Betonung der symbolischen Konstruktion sozialer Wirklichkeit die physischen und körperlichen Aspekte dieser Wirklichkeit in den Hintergrund, dennoch haben poststrukturalistische und diskurstheoretische Arbeiten unterschiedliche Perspektiven auf Materialität entwickelt. Ihr Fokus liegt dabei auf der Art und Weise, wie Materialität im Diskurs mit Bedeutung versehen und so zu einem Bestandteil symbolischer Prozesse wird. Die unterschiedlichen Perspektiven lassen sich wie folgt skizzieren:

1. Physisch-materielle Entitäten als Rohmaterial des Diskurses. Im Rahmen der Diskurstheorie von Laclau und Mouffe wird die Frage, wie Materialität mit Gesellschaft interagiert, über ihre Aufladung mit Bedeutung innerhalb des Diskurses erklärt. Die Existenz materieller Objekte und Ereignisse wird dabei nicht abgestritten, lediglich ihre Unabhängigkeit von diskursiven Ordnungen: ,What is denied is not that such objects exist externally to thought, but the rather different assertion that they could constitute themselves as objects outside any discursive condition of emergence" (Laclau und Mouffe, 2001, S. 108). Materialität wird also erst über den Diskurs zu einem Teil des Gesellschaftlichen, so dass kritische Erklärungen ebenfalls am Diskurs ansetzen müssen.

2. Materialität als unkontrollierbarer Rest. Dieser Sichtweise folgend gibt es eine vordiskursive, materielle Welt, die durch ihr „Hereinbrechen“, beispielsweise in Form von Naturkatastrophen oder anderen physischen Prozessen, den Diskurs destabilisiert und nach neuen symbolischen Deutungsweisen und Erklärungen verlangt (Zizek, 1992). Naturereignisse stellen demzufolge den (noch) nicht symbolisierten und kontrollierbaren 
„Rest“" dar, der erst durch die Aufladung mit Sinn gesellschaftlich verarbeitet wird (Stavrakakis, 1997). Die spezifischen und unterschiedlichen Funktionslogiken materieller Arrangements, beispielsweise von Ökosystemen, spielen dabei jedoch kaum eine Rolle.

3. Materialisierung von Diskursen. Viele poststrukturalistische Arbeiten verweisen darauf, dass Diskurse durch ihre materiellen Effekte „real“" werden. Die „Verkörperung“ und „Materialisierung“ von Diskursen zeigt sich beispielsweise in Sprechakten (Austin, 1972) sowie in Mustern raum- und körperbezogenen Agierens. Insbesondere feministische Theorien haben herausgearbeitet, wie sprachliche Zuschreibungen und gesellschaftliche Machtverhältnisse in ihrem Bezug auf Körper eine (physische) Realität erlangen (Butler, 1990, 2004; Strüver, 2003, 2005). Materialität ist in dieser Perspektive also eine notwendige Voraussetzung für die Realisierung des Diskurses, von dem eine ordnende und disziplinierende Macht ausgeht.

4. Materialität von Sprache. Pragmatisch-aussagenanalytische Ansätze der Diskursanalyse haben deutlich gemacht, dass der „Sinn“ gesprochener und geschriebener Sprache sich von den ProduzentInnen von Sprache nie vollständig kontrollieren lässt. Angermüller spricht in diesem Kontext von der ,opake[n] Materialität“ von Texten (2010), die dazu führt, dass Sinn immer das Produkt komplexer Verbindungen zwischen Text und Kontext ist, wobei diese Verbindungen ihrerseits durch formale Spuren im sprachlichen Material organisiert werden (Angermüller, 2007; Maingueneau, 1993; Mattissek, 2008).

Diese Skizze verdeutlicht, dass poststrukturalistische Ansätze, entgegen eines häufigen Missverständnisses, die Existenz einer außerdiskursiven bzw. vor-symbolischen Materialität durchaus anerkennen. $\mathrm{Zu}$ einem Teil gesellschaftlicher Wirklichkeit wird Materialität jedoch erst durch den Diskurs, der sie deutet, aneignet, diszipliniert und ordnet. Daher nehmen die spezifischen und unterschiedlichen Funktionsweisen, beispielsweise von Ökosystemen, menschlichen Körpern oder technischen Artefakten, höchstens eine nachgelagerte Rolle in Erklärungen gesellschaftlicher Machtverhältnisse und deren Veränderung ein.

Auf Michel Foucault trifft diese Aussage nur bedingt zu, denn in seinem Werk beanspruchen materielle Arrangements häufig eine zentrale Stellung (Philo, 2012). Insbesondere in seinen Arbeiten zur Gouvernementalität rückt Foucault materielle Aspekte sozialer Wirklichkeit bisweilen in den Vordergrund (Foucault, 2004; Deleuze, 1992). Dabei führt er auch den Begriff des Dispositiv ein, das er als ,ein entschieden heterogenes Ensemble, das Diskurse, Institutionen, architektonische Einrichtungen, reglementierende Entscheidungen, Gesetze, administrative Maßnahmen, wissenschaftliche Aussagen, philosophische, moralische oder philanthro- pische Lehrsätze, kurz: Gesagtes ebenso wohl wie Ungesagtes “ charakterisiert (Foucault, 1978, S. 119 f). In der Geographie wurde Foucault zunächst über den Diskursbegriff rezipiert, was auch die Lesart seiner Arbeiten zur Gouvernementalität beeinflusst hat. Das Dispositiv fand daher kaum Eingang in humangeographische Theoriedebatten, sicher auch, weil das Verhältnis von Diskurs, Dispositiv sowie materieller Wirklichkeit bei Foucault undeutlich bleibt.

Nimmt man die Erkenntnis diskurstheoretischer und poststrukturalistischer Arbeiten ernst, dass Materialität immer (auch) symbolisch verhandelt wird, so erscheint es wenig überzeugend, ausgehend vom Dispositiv einzelne Aspekte sozialer Wirklichkeit außerhalb des Diskurses - d.h. außerhalb sozialer Sinnsysteme - zu verorten, wie es beispielsweise Bührmann und Schneider (2008) vorschlagen. Vielversprechender ist es unseres Erachtens, das sowohl als auch einer ebenso symbolischen wie materiellen sozialen Wirklichkeit zu betonen, wie es Flache bzw. Relationale Ontologien tun (Castree, 2003; Marston et al., 2005). Dieses sowohl als auch kann jedoch nicht in einer bloßen Ergänzung der Betrachtung bestehen, einer Ergänzung, die dem Diskurs eine parallele Ordnung gegenüber stellt. Vielmehr geht es um eine Sensibilisierung für die vielfältigen, heterogenen und spannungsgeladenen Beziehungen, die sich in gesellschaftlichen Gefügen verdichten. Einen Ausgangspunkt für eine solche Perspektive bietet der Begriff des Agencements, den Deleuze und Guattari (1992) verwenden, und der nicht zuletzt auf Deleuzes Auseinandersetzung mit Foucaults Werk zurückgeht (Legg, 2011; Deleuze, 1992).

\section{Flache Ontologie und Assemblage-Theorie}

Theorien, die auf die Heterogenität gesellschaftlicher Wirklichkeiten verweisen, etablieren sich zunehmend in der humangeographischen Forschung (Whatmore, 2006; Bakker und Bridge, 2006; Braun, 2006). Gemeinsam ist ihnen das Ziel, Dualismen zwischen Gesellschaft und Natur zu überwinden, sie jedoch nicht auf ihre symbolische Aushandlung zu reduzieren (Latour, 1993). Stattdessen zeigen sie auf, wie Materielles (Biologisches, Physisches, Technisches etc.) und Symbolisches im Sozialen miteinander verwoben sind. Sie gründen insofern auf eine „Flache“ oder auch „Relationale Ontologie“, als sie die hierarchische Trennung zwischen Erkenntnis und Wirklichkeit, Materie und Sinn, Natur und Kultur aufheben und beiden Seiten der Dualismen den gleichen ontologischen Status beimessen (Castree, 2003; Escobar, 2007).

Wir möchten hier jenen Strang dieser Diskussionen aufgreifen, der sich auf die philosophischen Projekte von Gilles Deleuze und Felix Guattari bezieht (Deleuze und Guattari, 1992; Deleuze und Parnet, 1980) sowie auf Manuel DeLandas Arbeiten zu Assemblage-Theorie (DeLanda, 2011, 2006). Eine Flache Ontologie ist dabei gleichermaßen Ausdruck wie Grundlage einer Theorie, die Agencements (eng. 
Assemblage, dt. Gefüge) ${ }^{1}$ zum Ausgangspunkt gesellschaftswissenschaftlicher Untersuchungen macht. Die Möglichkeiten des Konzepts werden derzeit in unterschiedlichen humangeographischen Teilbereichen und Theoriedebatten eruiert, so zum Beispiel in der Wirtschaftsgeographie (Berndt und Boeckler, 2011) und Raumentwicklung (Van Wezemael und Loepfe, 2009), in der Politischen Geographie (Jeffrey et al., 2012; Lorimer, 2010), in der Gesellschaft-UmweltForschung (Braun, 2008) oder mit Blick auf die ScaleDebatte (Marston et al., 2005; Legg, 2009). Uns geht es an dieser Stelle insbesondere darum, durch die Begegnung von Assemblage-Theorie mit einem Machtbegriff im Sinne Foucaults das Spannungsverhältnis zwischen symbolischen und materiellen Aspekten gesellschaftlicher Gefüge einer geographischen Analyse zugänglich zu machen und die Verschiebungen aufzuzeigen, die sich ergeben, wenn man Gesellschaft-Umwelt-Verhältnisse unter dem Blickwinkel der Assemblage-Theorie betrachtet.

Als Agencement bzw. Gefüge bezeichnet Deleuze das Ergebnis von Verknüpfungen, beispielsweise von Individuen, Institutionen, Materien und Aussageregeln, die in ihrem $\mathrm{Zu}$ sammenwirken neue Eigenschaften und Fähigkeiten ausbilden (Deleuze und Parnet, 1980, S. 76). Soziale Entitäten, wie ein Staat oder ein Unternehmen, sind aus dieser Sicht also Gefüge, die auf historisch kontingente Prozesse des Verknüpfens zurückzuführen sind und deren (temporäre) Stabilität sich aus einer Regelhaftigkeit von Interaktionen ergibt: ,the identity of an assemblage should always be conceived as the product of a historical process, the process that brought its components together for the first time as well as the process that maintains its integrity through a regular interaction among its parts" (DeLanda, 2011, S. 185). Die Elemente, aus denen sich ein Gefüge zusammensetzt, sind dabei ihrerseits Gefüge, die jedoch nicht auf einen Funktionszusammenhang beschränkt bzw. durch diesen erschöpfend bestimmt sind. Gefüge können vielmehr in anderen Gefügen auf unterschiedlichen Maßstabsebenen mitwirken, so dass sie ein verzweigtes, nicht notwendig hierarchisch organisiertes Netz bilden: ein Individuum kann mit anderen Individuen interagieren und als Subjekt staatlicher Institutionen auftreten, ein Unternehmen ist Teil einer Warenkette und Schauplatz gesellschaftlicher Auseinandersetzungen.

Die Eigenschaften und Fähigkeiten von Gefügen sind emergent, das heißt sie übersteigen die Summe der Eigenschaften ihrer Teile, insofern sie erst im Zusammenwirken der Elemente des Gefüges entstehen (DeLanda, 2006). Erst in der Verknüpfung eines Walds mit Verarbeitungstechniken, Transportmöglichkeiten, Bewertungsverfahren etc. entsteht

\footnotetext{
${ }^{1}$ In englischsprachigen Theoriedebatten hat sich der Begriff Assemblage als Übersetzung für das frz. Agencement etabliert. Wir sprechen von Assemblage-Theorie, um den Bezug zur konzeptionellen Debatte hervorzuheben, nutzen jedoch den deutschen Begriff Gefüge für die Einheit der Analyse. Zum Problem der Übersetzung siehe auch Phillips (2006).
}

beispielsweise eine Forstwirtschaft, die spezifische Akteure und Wissensordnungen hervorbringt sowie Interaktionsprozesse zwischen Menschen und ihrer Umwelt strukturiert.

Während Gefüge durch ihre Eigenschaften charakterisiert werden können, verweisen ihre Fähigkeiten auf die Möglichkeit zur Interaktion. Die Fähigkeiten eines Gefüges, seine Handlungsfähigkeit oder Agens, sind dabei in einem doppelten Sinn relational: Zum einen sind sie Ausdruck der inneren Organisation des Gefüges und seiner emergenten Eigenschaften. Zum anderen werden Fähigkeiten erst in der Beziehung zu anderen Gefügen möglich, d.h. sie bedürfen der „Fähigkeit“ eines anderen Gefüges zu reagieren, affiziert zu werden, und aktualisieren sich daher erst in der Interaktion (DeLanda, 2011, S. 186). Da sich nie alle möglichen Begegnungen zwischen Gefügen vorhersagen lassen und da angesichts der Komplexität sozialer Gefüge kausale bzw. deterministische Interaktionen als Ausnahmefall zu betrachten sind, bilden Fähigkeiten einen prinzipiell unbegrenzten Möglichkeitsraum: die Potentiale einer neuen Technologie, beispielsweise, aktualisieren sich erst in (veränderlichen) Zusammenhängen, die nicht determiniert und der Technologie nicht immanent sind. Erst, wenn sie zu einem festen Bestandteil sozialer Gefüge wird, kann sie als Black Box Interaktionen stabilisieren, gleichermaßen jedoch auch zu einer Destabilisierung führen, wenn sie sich ,unerwartet" verhält (Latour, 1991, 1994). Gleichermaßen beruht die Stabilität von Gesellschaft-Umwelt-Gefügen auf einer „Ko-Operation“, auf einem „Zusammen-Wirken“ materieller und symbolischer Formationen. Veränderungen in materiellen Arrangements provozieren Verschiebungen im symbolischen Raum, ebenso wie symbolische Transformationen materielle „Effekte“ generieren, die häufig über linear-kausale bzw. deterministische Wirkungen hinausgehen und sich einer vollständigen Kontrolle und Vorhersehbarkeit entziehen.

Dem erkenntnistheoretischen Konstruktivismus diskurstheoretischer Arbeiten stellt die Assemblage-Theorie eine realistische Weltsicht gegenüber, in der Wirklichkeit als verzweigtes und offenes Geflecht von heterogenen Verbindungen erscheint. Wird die Kontingenz sozialer Wirklichkeit in poststrukturalistischen Ansätzen in der Regel auf die Unvollständigkeit symbolischer Ordnungen zurückgeführt - und damit weitgehend ohne Bezug auf materielle Prozesse erklärt - so ergibt sich Kontingenz in der Assemblage-Theorie aus der Komplexität der Wirklichkeit, aus den vielfältigen Möglichkeiten des In-Beziehung-Tretens von Gefügen. So kommt sie ohne die Annahme stabiler Identitäten oder Essenzen aus, da Eigenschaften und Fähigkeiten, beispielsweise von Staaten oder sozialen Bewegungen, von Märkten oder Individuen, auf historische Prozesse des Verknüpfens, Trennens und Ordnens zurückgeführt werden. Auf diesem Wege grenzt sich die Assemblage-Theorie von Ansätzen innerhalb der Geographie sowie der Sozial- und Politikwissenschaften ab, die Staat, Gesellschaft oder Kapitalismus zum a priori ihrer Analysen machen (Escobar, 2007, S. 107), ohne diese Kategorien auf ihre symbolische Aushandlung zu reduzieren. 
In der Assemblage-Theorie ist Sprache dennoch ein Bestandteil von Gefügen und ebenso ,wirklich“ wie Materialität. Mit Blick auf die Stabilisierung und den Wandel gesellschaftlicher Verhältnisse kommt ihr eine besondere Relevanz zu: sie verweist auf die Fähigkeit von Individuen, Institutionen und Gesellschaften sich auszudrücken, das heißt über symbolische Systeme in Interaktion zu treten. Wie sprachliche und materielle Teile in Gefügen zusammenwirken, soll im Folgenden näher beleuchtet werden.

\section{Sprache und Materialität}

Die Assemblage-Theorie verortet sprachliche wie nichtsprachliche Aspekte auf einer gemeinsamen ontologischen Ebene (Braun, 2008). Sprache und Materialität verweisen aus dieser Sicht auf zwei Dimensionen sozialer Wirklichkeit, die in Gefügen miteinander verschränkt sind, sich wechselseitig stützen, ohne dass zwischen beiden eine Gleichförmigkeit oder Hierarchie vorausgesetzt wird. Beide folgen unterschiedlichen, jedoch keineswegs unabhängigen Logiken. Sprache kommt mit Blick auf die Organisation von Gesellschaft eine besondere Rolle innerhalb der AssemblageTheorie zu: ,the effect of language on human behavior (as well as on the emergent behavior of communities and organizations) is so unique that it must be assigned its own parameter in an assemblage“ (DeLanda, 2011, S. 197). Trotz der unterschiedlichen erkenntnistheoretischen Perspektiven von Diskurs- und Assemblage-Theorie, können diskursanalytische Verfahren also durchaus einen Platz innerhalb der Assemblage-Theorie beanspruchen. Sprache markiert hier eine emergente Fähigkeit sozialer Gefüge zur Wissens- und Sinnproduktion. Die Gegenstände des Diskurses, sein zu ordnendes Feld und seine Machtwirkungen, sind aus Sicht der Assemblage-Theorie jedoch nicht primär auf eine symbolische Ordnung, sondern auf heterogene Gefüge ausgerichtet. Im Diskurs um den globalen Klimawandel wird beispielsweise „Wald“ zunehmend als Teil eines internationalen Emissionshandels konstruiert. Wie erfolgreich diese diskursive Verschiebung ist, hängt nicht zuletzt davon ab, wie erfolgreich die Kontrolle physisch-materieller Stoffflüsse und die Reorganisation der Interaktion von Mensch-Umwelt Gefügen im Sinne der Kohlenstoffspeicherung sind. Hier zeigt sich also, dass Materialität zwar auf einer diskursiven Ebene verhandelt wird, Stabilität und Wandel jedoch nicht allein auf dieser Ebene erklärbar sind.

Das Beispiel macht auch deutlich, dass Aussagen und ihre Gegenstände nicht in einem bloßen Repräsentationsverhältnis zueinander stehen. „Die Aussagen reduzieren sich nicht darauf, jeweils entsprechende Sachverhalte bloß zu beschreiben; beide stellen vielmehr so etwas wie nicht-parallele Formalisierungen dar" (Deleuze und Parnet, 1980, S. 77 f). ${ }^{2}$

\footnotetext{
${ }^{2}$ In seiner Arbeit über Foucault legt Deleuze nahe, dass sich die „Archäologie des Wissens“ (Foucault, 1981) durchaus bewusst auf diskursive Formationsregeln beschränkt und spätere Arbeiten Fou-
}

Aussagen und ihre Inhalte sind in Gefügen miteinander verflochten, sie setzen sich wechselseitig voraus und beeinflussen einander. Deleuze und Guattari sprechen daher von zwei Segmenten von Gefügen, einem Ausdruckssegment und einem Inhaltssegment. Sprache, als Ausdruckssegment, ist eine Besonderheit sozialer Gefüge. Sie ist performativ und insofern wirkmächtig, als sie auf die Organisation der Gegenstände, d.h. das Inhaltssegment eines Gefüges, einwirkt. Die Verurteilung eines Angeklagten findet beispielsweise im symbolischen Raum statt; von einem Richter ausgesprochen, setzt sie jedoch eine Reihe weiterer Veränderungen in Gang, die nicht auf das Symbolische beschränkt bleiben, wie das Abführen und Einsperren des Verurteilten. Da die Transformation innerhalb des Symbolischen stattfindet und damit zunächst unabhängig von den Körpern ist, auf die sie sich bezieht, sprechen Deleuze und Guattari auch von einer ,körperlosen Transformation" (Deleuze und Guattari, 1992, S. 113). Diese Fähigkeit ist der Sprache jedoch nicht immanent, sondern ergibt sich aus der Rolle, die ihr innerhalb eines bestimmten Gefüges zukommt. Die gleiche Aussage ist sinnlos, wird sie außerhalb des Gefüges Polizei-Gericht-Gefängnis geäußert, oder, wie Deleuze und Guattari an einem weiteren Beispiel verdeutlichen: „Wenn irgendjemand ruft ,Ich befehle die Generalmobilmachung', dann ist das eine kindische Aktion oder Schwachsinn; es ist nur dann ein Äußerungsakt, wenn es eine tatsächlich vorhandene Variable gibt, die das Recht zum Aussagen gibt“ (Deleuze und Guattari, 1992, S. 115). Diese Variablen, von denen Deleuze und Guattari sprechen und die einer Aussage ihre Macht verleihen, können dabei ebenso materiell wie symbolisch sein - und eine Veränderung dieser Möglichkeitsbedingungen provoziert umgekehrt Veränderungen in den Regeln der Aussagenproduktion.

Symbolische und materielle Aspekte sind im Gefüge also untrennbar miteinander verwoben, bedingen sich wechselseitig und wirken aufeinander ein, ohne sich jedoch zu determinieren. Stabilisierungen und Destabilisierungen können dabei innerhalb des Inhalts- oder Ausdruckssegments von Gefügen stattfinden, aber auch zwischen beiden. So wirkt sich der internationale Klimadiskurs auf Flüsse von Investitionen oder Kohlenstoff aus, doch diese Flüsse folgen ihrerseits spezifischen (ökonomischen oder biophysikalischen) Logiken, die unter Kontrolle gebracht werden müssen, um das Gefüge der Klimapolitik zu stabilisieren. Die Kontrollierbarkeit dieser Flüsse (die Speicherung von Treibhausgasen, das Verhalten von Investoren und Spekulanten) verweist also auf Bedingungen der Möglichkeit für Verhandlungen. Im zeitlichen Verlauf wird sichtbar, wie die Komponenten des Gefüges interagieren, sich verändern, zusammen funktionieren oder sich voneinander ,entfremden“.

caults diese dann um die Organisation von Sichtbarkeiten erweitert. In Deleuzes Lesart ist dies kein Widerspruch oder Bruch, sondern verweist auf unterschiedliche Dimensionen im Werk Foucaults, die sich im Dispositiv-Begriff berühren (Deleuze, 1992, S. 50 ff). Zum Verhältnis der Begriffe Dispositiv und Agencement siehe auch Legg (2011). 
Aus diesem Verständnis heraus lässt sich untersuchen, wie Ausdrucks- und Inhaltsformen zusammen- und aufeinander einwirken, in ein wechselseitiges Voraussetzungsverhältnis treten und sich destabilisieren. Die Assemblage-Theorie bietet damit einen theoretischen Rahmen an, der den Zwischenraum von symbolischen Ordnungen und ökologischen, technischen oder körperlichen Prozessen zu einem zentralen Schauplatz der Forschung werden lässt. Indem sie die besondere Rolle von Sprache betont, grenzt sie sich jedoch von der Akteurs-Netzwerk-Theorie ab, die häufig eine symmetrische Behandlung menschlicher und nicht-menschlicher Verknüpfungen fordert (Collins und Yearley, 1992; Murdoch, 1997). Gleichzeitig harmoniert sie mit der kritischen Haltung poststrukturalistischer Ansätze gegenüber starren und essentialistischen Erklärungen und ermöglicht ein Verständnis von Macht, das an Gouvernementalitätsstudien anschließt, jedoch die materiellen Facetten gesellschaftlicher Verhältnisse stärker betont und der Analyse zugänglich macht.

\section{Gesellschaftliche Machtverhältnisse}

Das Anliegen diskurstheoretischer und poststrukturalistischer Arbeiten lässt sich ganz allgemein als eine kritische Erklärung gesellschaftlicher Verhältnisse fassen. Welche Implikationen hat die Assemblage-Theorie für geographische Erklärungen? Zunächst erfordert die prinzipielle Offenheit der Assemblage-Theorie auch eine Offenheit im Forschungsprozess, um der Dynamik und Komplexität gesellschaftlicher Prozesse Rechnung zu tragen. Der Ansatz geht also mit einer spezifischen Haltung einher, ,an ethos of engagement that attends to the messiness and complexity of phenomena“ (Anderson et al., 2012). Die Art der Elemente und Verbindungen, die für eine wissenschaftliche Erklärung relevant werden, können daher nicht von vornherein feststehen. Die Kunst besteht darin, empirisch beobachtbaren Verbindungen zu folgen: die unterschiedlichen Netze zu kartieren und die Prozesse offenzulegen, die für die Stabilisierung und Veränderung sozialer Wirklichkeiten von Bedeutung sind. Es gilt, die heterogenen und teilweise widersprüchlichen Logiken herauszuarbeiten, die soziale Prozesse durchziehen. Dies impliziert auch eine Offenheit gegenüber unterschiedlichen Forschungsmethoden, die je nach Problemstellung zum Einsatz kommen. Wissenschaft selbst erscheint dann als ein Prozess des Verknüpfens und Interagierens, in dem Menschen, Beobachtungen und Gegenstände mit Theorien und Methoden in Berührung gebracht werden.

Des Weiteren geht die Betonung von Komplexität und Offenheit mit einem politischen Anspruch einher: durch das Aufzeigen der Komplexität und Kontingenz gesellschaftlicher Wirklichkeit werden Möglichkeiten zur Veränderung hervorgehoben und Machtverhältnisse als solche erkennbar. Die relationale Konzeption von Gefügen, die Betonung ihrer historischen „Gemachtheit“ und die Möglichkeit zum Wandel, sind dabei anschlussfähig an eine poststrukturalistische
Machtkonzeption im Sinne Foucaults, heben jedoch stärker ihre materiellen Aspekte und ihre Veränderlichkeit hervor (Philo, 2012). Die Assemblage-Theorie erweitert den Blick auf materielle Lebensbedingungen, praktisches Tun, die Anordnung von Körpern und auf dynamische ökologische Prozesse als immanente Bestandteile sozialer Wirklichkeit. Aus unserer Sicht scheinen mit Blick auf empirische Untersuchungen vier Facetten eines solchen ,re-materialisierten“ poststrukturalistischen Machtbegriffs besonders interessant:

1. Die Produktion von Wissen als Voraussetzung für Regierung. Hierbei spielen technische (Mess-)Apparate und ihre Funktionslogiken ebenso eine Rolle, wie Verfahren der Kalkulation, des Aggregierens oder Auswertens von Daten. Wissensproduktion markiert dabei Übergänge zwischen materiellen und symbolischen Dimensionen eines Gefüges. So entsteht Wissen beispielsweise in der Kombination neuer Technologien mit einer materiellen Umwelt und verweist auf veränderliche Möglichkeitsbedingungen des Einbezugs und der Organisation von Materialität in gesellschaftlichen Gefügen.

2. Regierungstechniken im Sinne Foucaults. Arbeiten zur Gouvernementalität verweisen auf Verfahren der Fremd- und Selbststeuerung, über die menschliche Praktiken auf der Mikro- und Makroebene entlang spezifischer Logiken bzw. Rationalitäten ausgerichtet werden. Diskursive Ordnungen spielen dabei eine zentrale Rolle, aber materielle Prozesse und Technologien sind ihrerseits Variablen, die neue Steuerungspotentiale hervorbringen und an der Transformation individueller Verhaltensweisen mitwirken.

3. Einen für die Geographie besonders relevanten Spezialfall von Regierungstechniken stellt das Arrangieren von menschlichen und nicht-menschlichen Praktiken im Raum dar. So verbinden sich beispielsweise in Raumplanung (Infrastrukturausbau, Abgrenzung von Baugebieten), in politischen Entscheidungen über Landnutzung (Ausweisung von Schutzgebieten, Förderung/Verbot bestimmter Nutzungspraktiken) oder in der Überwachung öffentlicher Räume symbolische und materielle Logiken und organisieren so Handlungspotentiale.

4. Handlungsfähigkeit bzw. Agens, d.h. die Möglichkeit in Interaktion $\mathrm{zu}$ treten und Veränderungen $\mathrm{zu}$ bewirken, ist aus Sicht der Assemblage-Theorie Ausdruck emergenter Eigenschaften und Fähigkeiten von Gefügen. Dieses stark relationale Verständnis von Agens erinnert an Foucaults Mikrophysik der Macht, die er im Zwischenraum gesellschaftlicher Interaktionen ansiedelt. Sie zeigt sich in Beziehungen, muss sich dort immer neu ,beweisen“ und ist somit nicht im Besitz einzelner Subjekte oder Institutionen (Foucault, 2005). Gegenüber diskurstheoretischen Ansätzen treten 
in der Assemblage-Theorie die materiellen Aspekte von Handlungspotentialen stärker in den Vordergrund.

Entlang dieser hier nur angedeuteten Linien lässt sich aus unserer Sicht die Einbindung materieller Aspekte sozialer Wirklichkeit in der Erklärung raumbezogener gesellschaftlicher Machtverhältnisse weiter verfolgen. Je nach Blickwinkel bieten sich dabei andere Schwerpunkte an und lassen sich weitere Facetten des Verhältnisses von Macht und Raum identifizieren. Im Folgenden werden wir einige der dargestellten theoretischen Überlegungen in Berührung mit einem Fallbeispiel aus dem Bereich der Gesellschaft-UmweltForschung bringen, um mögliche Verschiebungen zu verdeutlichen, die sich an die Assemblage-Theorie anschließen. Dieser empirische Fokus bietet sich an, weil in der Organisation von Gesellschaft-Umwelt-Verhältnissen die Verschränkung von materiellen und nicht-materiellen Aspekten gesellschaftlicher Wirklichkeit besonders deutlich zu Tage tritt.

\section{Waldpolitik in Thailand}

Waldpolitik spielt seit vielen Jahrzehnten in Thailand eine zentrale Rolle bei der Aushandlung gesellschaftlicher Verhältnisse. In zahlreichen aktuellen politischen Auseinandersetzungen im Land geht es um Zugangsrechte zu und Nutzungsweisen von natürlichen Ressourcen, und auch historisch war Waldpolitik von hoher Bedeutung, beispielsweise im Handel mit und in der Abgrenzung von Kolonialmächten. Begreift man Waldpolitik als Gefüge, so rückt in den Vordergrund, wie Diskurse (auf unterschiedlichen Maßstabsebenen) und physisch-materielle Prozesse interagieren, sich wechselseitig bedingen und transformieren. Mit dem oben skizzierten Ansatz lassen sich also zwei Fragen adressieren: Erstens, wie können Umbrüche in der thailändischen Waldpolitik erklärt werden und auf welche (symbolischen und materiellen) Verschiebungen sind Stabilisierungen und Destabilisierungen des Gefüges Waldpolitik zurückzuführen? Und zweitens, wie verändern sich Machtverhältnisse im Zusammenwirken materieller und symbolischer Logiken im Bereich der Waldpolitik?

Für das Gefüge der Waldpolitik gilt es also zu klären, wie zu unterschiedlichen Zeiten verschiedene (biologische, physikalische, technische, institutionelle, symbolische) Logiken zusammenwirken und dabei spezifische Nutzungsformen, Akteure und ökologische Prozesse miteinander in Beziehung setzen. Im Folgenden soll dies am Beispiel von drei Gegenstandsbereichen aufgezeigt werden, die für die derzeit ablaufenden Transformationen in der thailändischen Waldpolitik besonders einflussreich sind. Erstens treffen unterschiedliche Logiken in der Produktion von Wissensordnungen zusammen, da diese sowohl auf der Anwendung von Techniken (Statistiken, Messungen und Modellierungen, Fernerkundung), als auch auf den Funktionsweisen ökologischer Systeme sowie deren Verknüpfung mit bestimmten symbolischen Zuschreibungen (z. B. Wald als Ressource, als schützenswerte Natur oder als sakraler Ort) beruhen. Zweitens wird der Wald in unterschiedliche Nutzungssysteme und institutionelle Zusammenhänge integriert, wie beispielsweise in Gefüge des Naturschutzes oder des globalen Klimawandels, in denen jeweils rechtliche Systeme und institutionelle Vorgaben mit Praktiken der Nutzung und Inwertsetzung interagieren. Und schließlich spielen die Reaktionen und Prozesse innerhalb des Ökosystems „Wald“, in dem Bäume in einem wechselseitigen Konstitutionsverhältnis mit physikalischen, biotischen und abiotischen Prozessen stehen, eine zentrale Rolle für die Stabilisierung des Gefüges Waldpolitik. Wissensordnungen, Nutzungssysteme und institutionelle Gefüge sowie Prozesse innerhalb des Ökosystems Wald bedingen sich dabei wechselseitig und sind ihrerseits das Ergebnis des Zusammenwirkens sowohl symbolischer wie materieller Logiken. Daher können Veränderungen in einzelnen Dimensionen des Gefüges Waldpolitik zu einer Destabilisierung und Transformation des Gesamtgefüges führen.

\section{Waldpolitik im Spannungsfeld gesellschaftlicher Transformationen}

Waldpolitik in Thailand unterliegt, wie viele andere Formen der Organisation von Mensch-Umwelt-Verhältnissen, derzeit einem tiefgreifenden Wandel, der beispielsweise in Diskussionen um eine „Neoliberalisierung“ oder „Kommodifizierung" von Natur zum Ausdruck kommt (Bakker, 2005, 2009, 2010; McCarthy, 2005). Mensch-Umwelt-Verhältnisse, so die These dieser Arbeiten, werden zunehmend aus der Perspektive wirtschaftlicher Nützlichkeit beurteilt und nach den Logiken ökonomischer Effizienz „,gemanagt“. Beispiele hierfür sind die Debatte über „Ökosystemdienstleistungen“ (die Frage, welchen Nutzen bestimmte Ökosysteme für den Menschen erbringen und wie sich dieser Nutzen in Geld umrechnen lässt), ebenso wie der Versuch, den globalen Klimawandel über den Handel von $\mathrm{CO}_{2}$-Zertifikaten zu mindern. Bakker (2009, 2010), Bumpus (2011) und Robbins (2007) haben in diesem Zusammenhang deutlich gemacht, dass die derzeit beobachtbaren Umbrüche in der Organisation von Gesellschaft-Umwelt-Verhältnissen nicht allein durch einen diskursiv-symbolischen Wandel erklärt werden können. Vielmehr ermöglichen oder verhindern materielle Entitäten und deren inhärente Logiken bestimmte Aneignungsformen.

Mit Bezug auf Waldpolitik zeigen sich Tendenzen der Neoliberalisierung von Gesellschaft-Umwelt-Verhältnissen insbesondere in den Diskussionen um eine Einführung von REDD+ als Instrument des Klimaschutzes. REDD+ zählt zu den derzeit diskutierten flexiblen Mechanismen, die im Rahmen internationaler Klimaverhandlungen entwickelt werden. Ziel ist es, ärmeren Ländern einen finanziellen Ausgleich dafür zu zahlen, dass sie ihren Wald schützen - und damit potentielle Kohlenstoff-Emissionen verhindern (Bosetti und Rose, 2011; Clements, 2010). Dazu wird die gegenüber 
einem „Baseline“-Szenario eingesparte Menge an gespeichertem $\mathrm{CO}_{2}$ im Wald bestimmt und in einen Geldwert umgerechnet.

In der wissenschaftlichen Debatte um Potentiale und Grenzen der Einführung von REDD+ werden derzeit eine ganze Reihe offener Fragen der Implementierung und möglichen Effekte dieses Mechanismus diskutiert. Befürworter argumentieren, REDD+ habe das Potential, nicht nur Kohlenstoff-Emissionen zu verringern, sondern auch Armut zu bekämpfen und die Verwundbarkeit der ländlichen Bevölkerung zu verringern (Corbera et al., 2010, S. 359; Phelps et al., 2010, S. 325; Skutsch, 2011). Dementgegen stehen Befürchtungen, dass REDD+ negative Auswirkungen auf ländliche Bevölkerungsgruppen haben und bestehende Formen der Diskriminierung verstärken könnte (Ghazoul et al., 2010; Lawlor und Huberman, 2009, S. 271 ff; Skutsch und McCall, 2010, S. 398). Ein Argument der REDD-Kritiker ist dabei, dass REDD+ bestehende Formen einer Dezentralisierung von Wald-Governance unterminieren würde, da es Anreize für den Staat verstärke, die Kontrolle über Wald und damit die potentiellen Gewinne aus REDD+ für sich zu beanspruchen (Agrawal et al., 2010; Lewis, 2009, S. 567; Sandbrook et al., 2010, S. 332).

Eine der in diesem Kontext am prominentesten diskutierten Formen von Wald-Governance ist die community forestry, die insbesondere im Rahmen vieler Projekte der Entwicklungszusammenarbeit als Möglichkeit der Dezentralisierung propagiert wird ( $\mathrm{Li}, 2007)$. Als community forestry werden allgemein Nutzungsformen von Wald bezeichnet, bei denen Wald kollektiv, häufig von Dorfgemeinschaften, genutzt wird. Die Gemeinschaftswälder dienen unter anderem als Waldweide oder zum Sammeln von NichtholzForstprodukten (non timber forest products) für den Eigenbedarf und den Verkauf (Peluso et al., 1995). Heute praktizierte Formen von community forestry knüpfen einerseits an lange Traditionen der Waldnutzung an, sind aber gleichzeitig auch durch Verflechtungen, beispielsweise mit der internationalen Entwicklungszusammenarbeit oder - im Falle Thailands - mit nationalen politischen Programmen des Königshauses gekennzeichnet.

Vor dem Hintergrund dieser Debatten bietet die Assemblage-Theorie die Möglichkeit, bei der Frage nach den möglichen Konsequenzen einer Einführung von REDD+ zusätzliche Aspekte aufzuzeigen. Diese liegen, im Gegensatz zum Schwerpunkt der derzeitigen wissenschaftlichen Auseinandersetzung um REDD+, nicht allein im Bereich von Interessen, Akteurskonstellationen und institutionellen Verfasstheiten von Waldpolitik. Vielmehr geht es darum, die Neuverknüpfungen zwischen diskursiv-symbolischen und materiellen Aspekten von Waldnutzung und Waldpolitik sowie die daraus resultierenden Machteffekte aufzuzeigen. Aus dieser Perspektive führen die derzeitigen Anstrengungen zur Einführung von REDD+ dazu, dass im Zusammenwirken unterschiedlicher Komponenten - Stoffflüssen, diskursiven Repräsentationen, Messverfahren, institutionellen Settings - neue Akteure und Handlungspotentiale entstehen, die weder durch materielle Prozesse, noch einen Wandel diskursiver Repräsentationen allein zu erklären sind.

Im Folgenden eruieren wir einige Potentiale der Assemblage-Theorie am Beispiel derzeit ablaufender Umbrïche in der thailändischen Waldpolitik. Aus politischgeographischer Perspektive gehen wir insbesondere der Frage nach, wie sich im Übergang von einem Zustand des Gefüges (,Waldpolitik als Naturschutz“) zu einem anderen (,Waldpolitik als Instrument des Klimaschutzes“) gesellschaftliche Machtverhältnisse verschieben. Dies untersuchen wir exemplarisch am Beispiel von community forestry und der Frage, wie sich deren Status im Übergang von einem Naturschutz-Gefüge zu einem Klimaschutz-Gefüge verändert. ${ }^{3}$ Community forestry ist für die Untersuchung der Machteffekte unterschiedlicher Gefüge ein besonders spannendes Thema, da sich um diese Form der Waldnutzung in Thailand seit ca. zwei Jahrzehnten eine kontroverse Debatte entspinnt, die sowohl deren rechtlichen Status, wie auch deren de facto Akzeptanz betrifft (Usher, 2009).

\section{Waldpolitik als Naturschutz}

Nach einer Phase intensiver kommerzieller Waldrodung und daraus resultierender großflächiger Entwaldung und Walddegradation, trat in Thailand 1989 ein nationaler logging ban in Kraft, ein generelles Verbot der Abholzung von Wald mit Ausnahme von Plantagen (Vandergeest, 1996). Waldpolitik als Naturschutz beschreibt am besten den Charakter des Gefüges seit dem Erlass des logging ban. Kennzeichnend für dieses Gefüge ist auf der symbolisch-diskursiven Ebene eine strikte Trennung zwischen „Natur“ und „Kultur“: „Natur“ gilt es vor dem Menschen und seinen Einflüssen zu schützen, menschliche Nutzungen von Wald gelten als störend und „falsch“ (Laungaramsri, 2001; Wong et al., 2007). Waldpolitik zielt daher zunehmend darauf, die zunächst diskursive Trennung von Kultur und Natur in der Interaktion von Menschen und Wald umzusetzen. Sie markiert insofern eine „,körperlose Transformation“ im Sinne von Deleuze und Guattari, als der Diskurs des Naturschutzes eine Transformation der (materiell-räumlichen) Interaktionen von Menschen und Wald bedingt. Zugangsverbote und Vertreibungen als Mittel staatlicher Interventionen zielen darauf ab, die Bevölkerung aus den neu ausgewiesenen Schutzgebieten fernzuhalten.

Innerhalb dieses Naturschutz-Gefüges spielen die biotischen Prozesse des Waldes kaum eine Rolle: Staatliche

\footnotetext{
${ }^{3}$ Aus der Perspektive der Assemblage-Theorie kann community forestry selbst als Gefüge bzw. Assemblage beschrieben werden: als Verknüpfung alltäglicher Nutzungen, tradierter Wissensordnungen, biotischer Wachstumszyklen, ökologischer Prozesse und institutioneller Verknüpfungen (Li, 2007). An dieser Stelle geht es uns darum zu untersuchen, inwieweit das Gefüge der community forestry an zwei unterschiedliche Gefüge der thailändischen Waldpolitik anschlussfähig ist.
} 
Kontrolle des Wald-Mensch-Komplexes erfolgt einerseits per Fernerkundung, um gemäß einer 0/1-Logik zwischen beund entwaldeten Flächen zu unterscheiden, und andererseits durch die Überwachung und Disziplinierung der Bevölkerung, die über Nutzungsverbote und Umsiedlungserlasse von einer Nutzung des Waldes und seiner Umwandlung in landwirtschaftliche Nutzflächen abgehalten werden soll (Delang, 2005; Forsyth und Walker, 2008; Pye, 2005). Was für ein Wald nun tatsächlich innerhalb der Schutzgebiete wächst, ist hingegen weitgehend ohne Bedeutung. Insgesamt ist das Gefüge Waldpolitik als Naturschutz damit stärker auf die Kontrolle und Regierung von Menschen, als von Wald ausgelegt. Seine Machteffekte bestehen insbesondere in der $\mathrm{Zu}$ rückdrängung bestehender Formen der Nutzung von Wald, beispielsweise der shifting cultivation (Forsyth und Walker, 2008; Wong et al., 2007) oder der community forestry.

Für die community forestry innerhalb des NaturschutzGefüges lässt sich also festhalten, dass sie die Trennung in Mensch und Natur ,verletzt“, und daher eine prekäre und umstrittene Position einnimmt, die sich insbesondere in dem Verbot von community forestry in Schutzgebieten zeigt. Entsprechende staatliche Interventionen bleiben dabei nicht ohne Widerstand: Bereits seit den 1990er Jahren gibt es Proteste gegen die Diskriminierung von community forestry und (bislang erfolglose) Anstrengungen, deren Legalisierung zu erwirken (Laungaramsri, 2001; Usher, 2009).

\section{Waldpolitik als Instrument des Klimaschutzes}

Vor dem Hintergrund eines diskursiven Wandels von Gesellschaft-Umwelt-Verhältnissen, der die Nützlichkeit von Ökosystemen für den Menschen gemäß ökonomischer Logiken bewertet, setzt sich derzeit zunehmend eine Sichtweise auf Wald durch, die dessen Potential fokussiert, als $\mathrm{CO}_{2}$ Speicher die Folgen des anthropogenen Klimawandels abzumildern (Pistorius et al., 2012; Romain, 2012). Im Zusammenspiel von Erdsystemwissenschaften und Klimapolitik verfestigt sich die Verknüpfung globaler klimatischer Veränderungen mit regionalen biologischen Veränderungen (Wachstum bzw. Degradierung von Wald) und wird zur Grundlage für eine Reorganisation von Institutionen, die auch neue Mess- und Kontrolltechnologien bedingt. Das Beispiel der thailändischen Waldpolitik zeigt, wie sich in diesem Zusammenhang Machtverhältnisse verschieben, insbesondere mit Blick auf community forestry.

Das Wissen über globale Klimaveränderungen, die institutionalisierten Aushandlungsprozesse der Klimapolitik und die damit verknüpfte Problematisierung von Entwaldung und Walddegradation, führen zu Transformationen im Gefüge der thailändischen Waldpolitik: Naturschutz wird dabei zunehmend abgelöst durch Waldpolitik als Instrument des Klimaschutzes. Dieses veränderte Gefüge verknüpft die bio-physikalischen Eigenschaften der Kohlenstoffspeicherung von Wald mit diskursiven Repräsentationen von Wald als Kohlenstoffsenke. Diese Verknüpfung basiert auch auf der Implementierung neuer Messmethoden und Modelle, die es ermöglichen, die Interaktionen zwischen Waldnutzung und Klimaemissionen zu quantifizieren. Zu diesen Verfahren gehören die Kartierung unterschiedlicher Spezies, die Anwendung biologischer und forstwirtschaftlicher Wachstumsmodelle sowie biophysikalische Verfahren zur Bestimmung der in einem Waldabschnitt gespeicherten Menge an Kohlenstoff. Sie heben damit dezidiert auf die Qualität und Art des Waldes ab und berücksichtigen, sehr viel stärker als die bislang im Kontext der thailändischen Forstpolitik etablierten Fernerkundungsverfahren, die biotischen Eigenschaften des Waldes (Interview Asian Development Bank, Thailand, 10 Februar 2012).

Diese Verknüpfung von Waldnutzung und internationalem Klimadiskurs ermöglicht eine Neuausrichtung von Waldpolitik, die sich in vielerorts entstehenden REDD+Pilotprojekten manifestiert und darauf abzielt, Interaktionen zwischen Menschen und Wald in Bezug auf dessen Kohlenstoffspeicherkapazitäten zu optimieren. Waldpolitik wird damit zum Gegenstand biopolitischer Interventionen im Foucaultschen Sinne: Ziel ist es, sowohl (globale) Waldbestände als auch (nationale und individuelle) Waldnutzungspraktiken so $\mathrm{zu}$ ordnen, dass die globalen $\mathrm{CO}_{2}$-Konzentrationen stabilisiert werden, um die „Gesundheit“ der Weltbevölkerung zu gewährleisten. Im Gegensatz zum Naturschutz-Gefüge steht hier also nicht die Trennung, sondern vielmehr die aktive Steuerung der Interaktionen zwischen Mensch und Umwelt im Fokus. Entsprechend rücken Formen der politischen Steuerung in den Vordergrund, die über die Anleitung der Bevölkerung zu möglichst klima-effizienten Formen der Waldnutzung das Verhältnis von Mensch und Wald zu optimieren suchen. Damit werden für eine Analyse von Waldpolitik - auch aus politisch-geographischer Perspektive - Erkenntnisse der Forstwirtschaft und Vegetationsgeographie relevant, die hier bislang kaum eine Rolle spielten.

Exemplarisch dafür, welche Rolle Materialität in der Neuformierung von Waldpolitik spielt, steht die Neubewertung von community forestry, bei der naturwissenschaftliche Analysen eine zentrale Rolle einnehmen. Auf der diskursiven Ebene wird deutlich, dass mit dem Wandel der Funktion von Wald hin zu einem Kohlenstoffspeicher, die Legitimationsbasis für ein Verbot von community forestry geschwächt wird, denn das veränderte Gefüge gründet nicht mehr auf einer kategorischen Trennung zwischen Mensch und Natur. Es entstehen neue Handlungspotentiale in der Interaktion zwischen menschlichen Praktiken, Technologien und Biosphäre, die auch zu einer Neubewertung der Stellung von community forestry in Thailand führen. Diese Stellung wird zunehmend durch die Anschlussfähigkeit von community forestry an klimabezogene Waldpolitik bestimmt.

Derzeit laufende Projekte zum capacity building in potentiellen REDD+ Teilnehmerländern, in deren Rahmen die notwendigen institutionellen Rahmenbedingungen für eine Einführung von REDD+ geschaffen werden sollen, sowie 
Erfahrungen aus der Durchführung von Pilotprojekten und -studien zeigen, dass für diese Anschlussfähigkeit vor allem zwei Typen von Beziehungen maßgeblich sind: Auf der diskursiven Ebene argumentieren Vertreter von pro people-Organisationen und Entwicklungszusammenarbeit, dass community forestry im Rahmen der Implementierung von REDD+ geschützt und gestärkt werden muss, um die sozialen und partizipationsbezogenen UN-Standards zur Berücksichtigung der Interessen lokaler Bevölkerungsgruppen zu erfüllen (IWGIA und AIPP, 2011). Auf einer zweiten Ebene kommen darüber hinaus aber sehr viel stärker auch die materiellen Austauschbeziehungen und deren Messung mit Hilfe unterschiedlicher Verfahren zum Tragen.

Insbesondere rückt die Frage in den Blick, wie viel Kohlenstoff in Flächen gespeichert wird, die im Rahmen von community forestry genutzt werden. Einige Studien legen nahe, dass die praktizierten Formen nachhaltiger Waldnutzung bei richtiger Durchführung in der Lage sind, die gespeicherte Kohlenstoffmenge nicht nur konstant zu halten, sondern darüber hinaus Degradation zu verringern und damit die Speicherkapazität zu erhöhen (K:TGAL, 2009; Northern Development Foundation et al., 2011; Skutsch und Solis, 2010). Daraus leitet sich die Forderung an die Klimapolitik ab, community forest management als Strategie zur Mitigation von Klimawandel ernst zu nehmen und zu fördern und Dorfgemeinschaften zu einer möglichst ,effektiven“ Nutzung im Sinne einer negativen Emissionsbilanz anzuleiten (Skutsch und Solis, 2010). Arbeiten am Schnittfeld zwischen Landschaftsökologie, Forstwissenschaft und Vegetationsgeographie einerseits und sozialwissenschaftlichen Ansätzen andererseits könnten hier die Möglichkeiten und (ökologischen wie sozialen) Auswirkungen verschiedener Nutzungsformen näher untersuchen.

Wenngleich sich durch diese Neuausrichtung von Waldpolitik neue Anschlussmöglichkeiten und Legitimationen für das community forestry-Gefüge ergeben, sind diese doch gleichzeitig durch erhebliche Unterschiede in den Formen der Wissensproduktion zwischen Klimapolitik einerseits sowie Subsistenz- und bestehenden Nutzungspraktiken andererseits gekennzeichnet. Während die Instrumente und Techniken der Wissensproduktion in der Klimapolitik auf der Anwendung von MRV-Verfahren (Monitoring, Reporting, Verification) beruhen und auf Vereinheitlichung und Nachvollziehbarkeit abzielen, sind alltägliche Nutzungspraktiken durch weit geringere Kodifizierung und Standardisierung gekennzeichnet (Lovell und Liverman, 2010). Die Zukunft der community forestry wird damit zunehmend abhängig von ihrer „Kompatibilität“ mit den Kriterien und Steuerungsmechanismen der internationalen Klimapolitik. Ziel einer vertiefenden wissenschaftlichen Analyse könnte es vor diesem Hintergrund sein, mögliche Konvergenzen, Anschlussmöglichkeiten oder Widersprüche herauszuarbeiten, die sich aus diesen unterschiedlichen Formen der Produktion und Regulierung von Wissen und Nutzungspraktiken ergeben.
Das Beispiel der Veränderung von Waldpolitik in Thailand, die in ähnlicher Form derzeit auch in anderen tropischen Ländern abläuft, deutet an, wie sich Machtverhältnisse im Zusammenspiel symbolisch-diskursiver und physischmaterieller Prozesse und deren (Neu-)Verknüpfung innerhalb eines Gefüges der Waldpolitik als Instrument des Klimaschutzes transformieren. Dabei wird insbesondere die Legitimität etablierter Formen der subsistenzorientierten Waldnutzung und damit die Stabilität und gesellschaftliche Stellung dieser Nutzungspraktiken neu verhandelt. Diese Neuverhandlung beruht nicht zuletzt auf der "Kooperation" der biotischen Komponenten des Gefüges (Steigerung bzw. Stabilisierung der Kohlenstoffspeicherkapazität von Wald), wobei die Anschlussfähigkeit an das Gefüge der Klimapolitik über Technologien der Messung der biophysikalischen Eigenschaften von Wäldern gewährleistet wird. Damit wird deutlich, dass Materialität auf mehrfache Art und Weise an der Konstitution gesellschaftlicher Machtverhältnisse beteiligt ist: Erstens über die Produktion von Wissen, welches an (materielle) Technologien und deren Möglichkeiten der Interaktion mit Aspekten der physisch-materiellen Umwelt gebunden ist; zweitens über Regierungstechniken, die an der Interaktion zwischen Mensch und Umwelt ansetzen und zum Ziel haben, die Kohlenstoffspeicherkapazitäten von MenschUmwelt-Gefügen zu optimieren; und drittens über die Veränderung und Neubewertung möglicher Interaktionen zwischen Menschen und ihrer (physischen) Umwelt, die sich aus den Verknüpfungen von materiellen und symbolischen Elementen ergeben.

\section{Fazit}

Ausgehend von jüngeren Entwicklungen in der Humangeographie, die heterogene Gefüge zum Ausgangspunkt wissenschaftlicher Erklärungen machen, haben wir einige Linien skizziert, entlang derer sich das Verhältnis von Geographie, Macht und Gesellschaft denken lässt. Erkennt man an, dass materielle und diskursive Ordnungen in sozialen Gefügen untrennbar ineinander verschränkt sind, dann erfordern kritische Erklärungen gesellschaftlichen Wandels einen Blick auf die wechselseitigen Voraussetzungen zwischen Materiellem und Symbolischem sowie ein Verständnis der heterogenen und komplexen Logiken, die soziale Prozesse durchziehen. Eine Geographie, gedacht entlang der Linien einer Assemblage-Theorie, vermag dann die materiellen Aspekte von Machtverhältnissen stärker hervorzuheben, als es diskurstheoretische Ansätze häufig tun. Das heißt jedoch nicht, dass die Erkenntnisse aus diskurstheoretischen und poststrukturalistischen Ansätzen einem idealisierten Symmetrieprinzip zum Opfer fallen müssen. Ein Verständnis diskursiver Aushandlungsprozesse ist für kritische Erklärungen gesellschaftlichen Wandels ebenso unabdingbar, wie ein Verständnis der Dynamik materieller Prozesse und der Untrennbarkeit menschlicher und nicht-menschlicher 
Gefüge. Einer Rematerialisierung human- und politischgeographischer Forschung kann es also nicht darum gehen, bisherige Erkenntnisse aus diskurstheoretischen Arbeiten zu verwerfen. Vielversprechender erscheint es, die emergenten Möglichkeiten sozialer Gefüge zur symbolischen Organisation von Verknüpfungen herauszustellen. Deleuze und Guattari bieten hier eine Perspektive an, die den Zwischenraum symbolischer und materieller Ordnungen in ihrer wechselseitigen Bedingtheit ausleuchtet, ohne dabei in ein deterministisches Verständnis ihrer Interaktionen zurückzufallen.

Die Analyse des Fallbeispiels hat gezeigt, dass eine solche Perspektive nicht nur eine größere Offenheit sozialwissenschaftlicher Forschung gegenüber materiellen Verhältnissen und Prozessen erfordert, sondern auch neue Anknüpfungspunkte zu naturwissenschaftlichen Methoden und Erkenntnissen schafft. Gerade einem Fach wie der Geographie, in dem die Möglichkeiten der Interaktion zwischen physisch- und humangeographischen Perspektiven ein Dauerbrenner fachstrategischer Diskussionen ist, eröffnen sich hier Räume für eine Zusammenarbeit, bei der naturwissenschaftliche Perspektiven einen wichtigen Beitrag zu kritischen Erklärungen gesellschaftlicher Machtverhältnisse leisten können.

Edited by: B. Korf

Reviewed by: three anonymous referees

\section{Literatur}

Agrawal, A., Nelson, F., Adams, W. M., and Sandbrook, C.: Governance and REDD: a reply to Wunder, Oryx, 44, 337-338, 2010.

Anderson, B., Kearnes, M., McFarlane, C., and Swanton, D.: On assemblages and geography, Dialogues in Human Geography, 2, 171-189, doi:10.1177/2043820612449261, 2012.

Angermüller, J.: Nach dem Strukturalismus; Theoriediskurs und intellektuelles Feld in Frankreich, Transcript, Bielefeld, 2007.

Angermüller, J.: Widerspenstiger Sinn. Skizze eines diskursanalytischen Forschungsprogramms nach dem Strukturalismus, in: Diskursanalyse meets Gouvernementalitätsforschung, edited by: Angermüller, J. and Van Dyk, S., Campus, Frankfurt am Main, 71-100, 2010.

Austin, J.: Zur Theorie der Sprechakte. Deutsche Bearbeitung von Eike von Savigny, Reclam, Stuttgart, 1972.

Bakker, K.: Neoliberalizing nature? Market environmentalism in water supply in England and Wales, Ann. Assoc. Am. Geogr., 95, 542-565, doi:10.1111/j.1467-8306.2005.00474.x, 2005.

Bakker, K. and Bridge, G.: Material worlds? Resource geographies and the "matter of nature", Prog. Hum. Geogr., 30, 5-27, doi:10.1191/0309132506ph588oa, 2006.

Bakker, K.: Neoliberal nature, ecological fixes, and the pitfalls of comparative research, Environ. Plann. A, 41, 1781-1787, doi:10.1068/a4277, 2009.

Bakker, K.: The limits of "neoliberal natures": Debating green neoliberalism, Prog. Hum. Geogr., 34, 715-735, doi:10.1177/0309132510376849, 2010.
Berndt, C. and Boeckler, M.: Geographies of markets: Materials, morals and monsters in motion, Prog. Hum. Geogr., 35, 559-567, doi:10.1177/0309132510384498, 2011.

Bosetti, V. und Rose, S. K.: Reducing carbon emissions from deforestation and forest degradation: issues for policy design and implementation, Environ. Dev. Econ., 16, 357-360, doi:10.1017/s1355770x11000143, 2011.

Braun, B.: Environmental issues: global natures in the space of assemblage, Prog. Hum. Geogr., 30, 644-654, doi:10.1177/0309132506070180, 2006.

Braun, B.: Environmental issues: inventive life, Prog. Hum. Geogr., 32, 667-679, doi:10.1177/0309132507088030, 2008.

Bührmann, A. und Schneider, W.: Vom Diskurs zum Dispositiv: Eine Einführung in die Dispositivanalyse, Transcript, Bielefeld, 2008.

Bumpus, A. G.: The Matter of Carbon: Understanding the Materiality of $\mathrm{tCO}(2) \mathrm{e}$ in Carbon Offsets, Antipode, 43, 612-638, doi:10.1111/j.1467-8330.2011.00879.x, 2011.

Butler, J.: Gender trouble, 1. publ. ed., Feminism and the subversion of identity, Routledge, New York, 1990.

Butler, J.: Undoing Gender, Routledge, New York, 2004.

Castree, N.: Environmental issues: relational ontologies and hybrid politics, Prog. Hum. Geogr., 27, 203-211, doi:10.1191/0309132503ph422pr, 2003.

Clements, T.: Reduced Expectations: the political and institutional challenges of REDD+, Oryx, 44, 309-310, doi:10.1017/s0030605310000712, 2010.

Collins, H. M. and Yearley, S.: Epistemological Chicken, in: Science as Practice and Culture, edited by: Pickering, A., The University of Chicago Press, Chicago, 301-326, 1992.

Corbera, E., Estrada, M., and Brown, K.: Reducing greenhouse gas emissions from deforestation and forest degradation in developing countries: revisiting the assumptions, Climatic Change, 100, 355-388, doi:10.1007/s10584-009-9773-1, 2010.

DeLanda, M.: A New Philosophy of Society: Assemblage Theory and Social Complexity, Continuum, London, New York, 2006.

DeLanda, M.: Philosophy and Simulation: The Emergence of Synthetic Reason, Continuum, London, New York, 2011.

Delang, C. O.: The political ecology of deforestation in Thailand 1840-1990s, Geography, 90, 225-237, 2005.

Deleuze, G. and Parnet, C.: Dialoge, Suhrkamp, Frankfurt am Main, 1980.

Deleuze, G.: Foucault, Suhrkamp, Frankfurt am Main, 1992

Deleuze, G. and Guattari, F.: Tausend Plateaus: Kapitalismus und Schizophrenie, Merve, Berlin, 1992.

Escobar, A.: The "ontological turn" in social theory. A Commentary on "Human geography without scale", by Sallie Marston, John Paul Jones II and Keith Woodward, T. I. Brit. Geogr., 32, 106111, doi:10.1111/j.1475-5661.2007.00243.x, 2007.

Forsyth, T. and Walker, A.: Forest guardians, forest destroyers: the politics of environmental knowledge in Northern Thailand, University of Washington Press, Seattle, 2008.

Foucault, M.: Dispositive der Macht. Über Sexualität, Wissen und Wahrheit, Merve, Berlin, 1978.

Foucault, M.: Archäologie des Wissens, Suhrkamp, Berlin, 1981.

Foucault, M.: Sicherheit, Territorium, Bevölkerung. Vorlesung am Collège de France 1977-1978, Suhrkamp, Frankfurt am Main, 2004. 
Foucault, M.: Analytik der Macht, Suhrkamp, Frankfurt am Main, 2005.

Ghazoul, J., Butler, R. A., Mateo-Vega, J., und Koh, L. P.: REDD: a reckoning of environment and development implications, Trends in ecology \& evolution, 25, 396-402, doi:10.1016/j.tree.2010.03.005, 2010.

Glasze, G. and Mattissek, A.: Handbuch Diskurs und Raum, Transcript, Bielefeld, 2009.

Glynos, J. and Howarth, D.: Logics of Critical Explanation in Social and Political Theory, Routledge, Abingdon, New York, 2007.

IWGIA und AIPP: Understanding community-based REDD+. A manual for indigenous communities, International Work Group for Indigenous Affairs, Asia Indigenous Peoples Pact, Chiang Mai, 2011.

Jackson, P.: Rematerializing social and cultural geography, Social \& Cultural Geography, 1, 9-14, doi:10.1080/14649369950133449, 2000.

Jeffrey, A., McFarlane, C., and Vasudevan, A.: Rethinking Enclosure: Space, Subjectivity and the Commons, Antipode, 44, 12471267, doi:10.1111/j.1467-8330.2011.00954.x, 2012.

Kazig, R. and Weichhart, P.: Die Neuthematisierung der materiellen Welt in der Humangeographie, Berichte zur deutschen Landeskunde, 83, 109-128, 2009.

Keller, R., Hirseland, A., Schneider, W., and Viehöver, W. (Eds.): Handbuch Sozialwissenschaftliche Diskursanalyse, dritte erweiterte Auflage, VS Verlag für Sozialwissenschaften, Wiesbaden, 2011.

Laclau, E. and Mouffe, C.: Hegemony and Socialist Strategy: Towards a Radical Democratic Project, Second Edition, Verso, London, New York, 2001.

Latour, B.: Technology is society made durable, in: A Sociology of Monsters: Essays on Power, Technology, and Domination, edited by: Law, J., Routledge, London, 103-131, 1991.

Latour, B.: We have never been modern, Harvester Wheatsheaf, New York, IX, 1993.

Latour, B.: On Technical Mediation: Philosophy, Sociology, Genealogy, Common Knowledge, 3, 29-64, 1994.

Laungaramsri, P.: Redefining Nature: Karen Ecological Knowledge and the Challenge to the Modern Conservation Paradigm, Earthworm Books, Chennai, 2001.

Lawlor, K. and Huberman, D.: Reduced emissions from deforestation and forest degradation (REDD) and human rights, in: Rightsbased approaches: Exploring issues and opportunities for conservation, edited by: Campese, J., Sunderland, T. C. H., Greiber, T., and Oviedo, G., Center for International Forestry Research (CIFOR) and The International Union for Conservation of Nature (IUCN), Bogor, Indonesia, 269-285, 2009.

Legg, S.: Of scales, networks and assemblages: The league of nations apparatus and the scalar sovereignty of the government of India, T. I. Brit. Geogr., 34, 234-253, doi:10.1111/j.14755661.2009.00338.x, 2009.

Legg, S.: Assemblage/apparatus: using Deleuze and Foucault, Area, 43, 128-133, doi:10.1111/j.1475-4762.2011.01010.x, 2011.

Lewis, S. L.: Carbon emissions: the poorest forest dwellers could suffer, Nature, 462, doi:10.1038/462567a, 2009.

Li, T. M.: Practices of Assemblage and Community Forest Management, Econ. Soc., 36, 263-293, doi:10.1080/03085140701254308, 2007.
Lorimer, J.: International conservation "volunteering" and the geographies of global environmental citizenship, Polit. Geogr., 29, 311-322, 2010.

Lovell, H. and Liverman, D.: Understanding Carbon Offset Technologies, New Polit. Econ., 15, 255-273, doi:10.1080/13563460903548699, 2010.

Maingueneau, D.: Le Contexte de l'oeuvre littéraire, Paris 1993.

Marston, S. A., Jones, J. P., and Woodward, K.: Human geography without scale, T. I. Brit. Geogr., 30, 416-432, doi:10.1111/j.1475-5661.2005.00180.x, 2005.

Mattissek, A.: Die neoliberale Stadt. Diskursive Repräsentationen im Stadtmarketing deutscher Großstädte, Transcript, Bielefeld, 2008.

McCarthy, J.: Devolution in the woods: community forestry as hybrid neoliberalism, Environ. Plann. A, 37, 995-1014, doi:10.1068/a36266, 2005.

Murdoch, J.: Inhuman/nonhuman/human: actor-network theory and the prospects for a nondualistic and symmetrical perspective on nature and society, Environ. Plann. D, 15, 731-756, 1997.

Northern Development Foundation, Huay Hin Lad community, and Oxfam-GB: Climate change, trees and livelihoods: a case study on the carbon footprint of a Karen community in Northern Thailand, Chiang Mai, 2011.

Peluso, N. L., Vandergeest, P., and Potter, L.: Social Aspects of Forestry in Southeast Asia: A Review of Postwar Trends in the Scholarly Literature, Journal of Southeast Asian Studies, 26, 196-218, 1995.

Phelps, J., Guerrero, M. C., Dalabajan, D. A., Young, B., and Webb, E. L.: What makes a "REDD" country?, Global Environmental Change, 20, 322-332, doi:10.1016/j.gloenvcha.2010.01.002, 2010.

Phillips, J.: Agencement/Assemblage, Theory, Culture \& Society, 23, 108-109, doi:10.1177/026327640602300219, 2006.

Philo, C.: More words, more worlds: reflections on the cultural turn and human geography, in: Cultural turns/geographical turns: perspectives on cultural geography, edited by: Cook, I., Crouch, D., Naylor, S., and Ryan, J., Prentice Hall, Harlow, 26-53, 2000.

Philo, C.: A "new Foucault" with lively implications - or "the crawfish advances sideways", T. I. Brit. Geogr., 37, 496-514, doi:10.1111/j.1475-5661.2011.00484.x, 2012.

Pistorius, T., Schaich, H., Winkel, G., Plieninger, T., Bieling, C., Konold, W., and Volz, K.-R.: Lessons for REDDplus: A comparative analysis of the German discourse on forest functions and the global ecosystem services debate, Forest Policy and Economics, 18, 4-12, doi:10.1016/j.forpol.2011.09.001, 2012.

Pye, O.: Khor Jor Kor, Studies in Contemporary Thailand 14, White Lotus, Bangkok, 2005.

Robbins, P.: Nature talks back: studying the economic life of things, in: Politics and Practice in Economic Geography, edited by: Tickell, A., Sheppard, E., Peck, J., and Barnes, T., Sage, London, Thousand Oaks, New Delhi, Singapore, 49-59, 2007.

Romain, P.: Payments for Environmental Services (PES) in the public policy landscape: "Mandatory" spices in the Indonesian recipe, Forest Policy and Economics, 18, 23-29, doi:10.1016/j.forpol.2011.09.002, 2012.

Sandbrook, C., Nelson, F., Adams, W. M., and Agrawal, A.: Carbon, forests and the REDD paradox, Oryx, 44, 330-334, doi:10.1017/s0030605310000475, 2010. 
Skutsch, M. and Solis, S.: How much carbon does community forestry management save?, in: Community forest monitoring for the carbon market, edited by: Skutsch, M., Earthscan, London, Washington DC, 16-30, 2010.

Skutsch, M. M. and McCall, M. K.: Reassessing REDD: governance, markets and the hype cycle, Climatic Change, 100, 395402, doi:10.1007/s10584-009-9768-y, 2010.

Skutsch, M. M.: Why community forest monitoring?, in: Community forest monitoring for the carbon market: opportunities under REDD, Earthscan, London, 2011.

Stavrakakis, Y.: Green Fantasy and the Real of Nature: Elements of a Lacanian Critique of Green Ideological Discourse, Journal for the Psychoanalysis of Culture \& Society, 2, 123-132, 1997.

Strüver, A.: „Das duale System“: Wer bin ich - und wenn ja, wie viele? Identitätskonstruktionen aus feministischpoststrukturalistischer Perspektive, in: Kulturgeographie. Aktuelle Ansätze und Entwicklungen, edited by: Gebhardt, H., Reuber, P., and Wolkersdorfer, G., Spektrum, Heidelberg, 113-128, 2003.

Strüver, A.: Macht Körper Wissen Raum? Ansätze für eine Geographie der Differenzen, Beiträge zur Bevölkerungs- und Sozialgeographie, Bd. 9, Wien, 2005.
Usher, A. D.: Thai forestry. A critical history, Silkworm Books, Chiang Mai, 2009.

Wezemael, J. and Loepfe, M.: Veränderte Prozesse der Entscheidungsfindung in der Raumentwicklung, Geogr. Helv., 64, 106118, doi:10.5194/gh-64-106-2009, 2009.

Vandergeest, P.: Mapping nature: Territorialization of forest rights in Thailand, Society \& Natural Resources, 9, 159-175, doi:10.1080/08941929609380962, 1996.

Whatmore, S.: Materialist returns: practising cultural geography in and for a more-than-human world, Cultural Geographies, 13, 600-609, doi:10.1191/1474474006cgj377oa, 2006.

Wong, T., Delang, C. O., and Schmidt-Vogt, D.: What is a forest? Competing meanings and the politics of forest classification in Thung Yai Naresuan Wildlife Sanctuary, Thailand, Geoforum, 38, 643-654, doi:10.1016/j.geoforum.2006.11.014, 2007.

Zizek, S.: Looking Awry. An introduction to Jacques Lacan through Popular Culture, MIT Press, Cambridge, 1992. 